\title{
THE Hierarchical Algorithm FOR DETECTING ROAD MARKING LINES
}

\author{
Makagonov Nikita, Bakhshiev Aleksandr, Semakova Anna
}
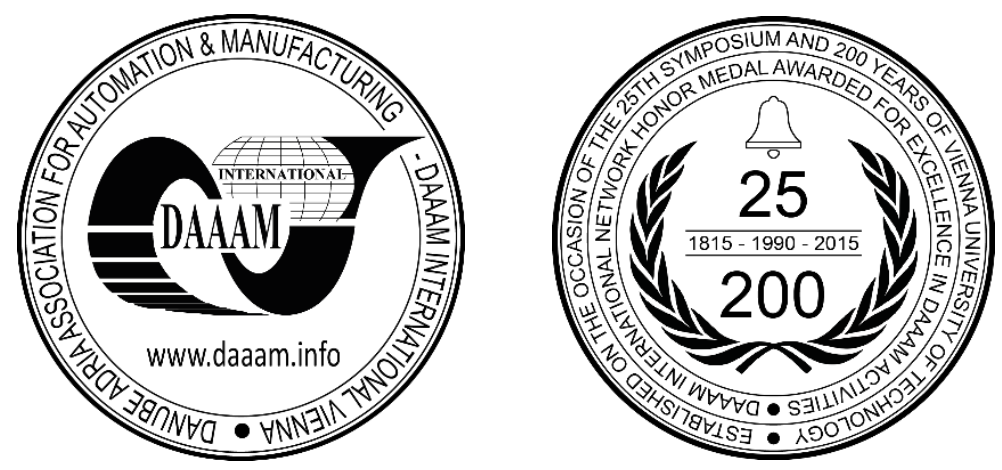

This Publication has to be referred as: Makagonov, N[ikita]; Bakhshiev, A[leksandr] \& Semakova, A[nna] (2018). The Hierarchical Algorithm for Detecting Road Marking Lines, Proceedings of the 29th DAAAM International Symposium, pp.1168-1172, B. Katalinic (Ed.), Published by DAAAM International, ISBN 978-3-902734-20-4, ISSN 1726-9679, Vienna, Austria

DOI: $10.2507 / 29$ th.daaam.proceedings. 168

\begin{abstract}
This paper is dedicated to the hierarchical algorithm for detecting road marking lines for autonomous cars. The main idea of this road marking recognition method is sequential filtering of image areas, which are fragments of the road marking with certain probability. The process of the markup recognition is divided into 4 consecutive stages. The paper presents three methods for first stages, that are used parallel, and methods that are used on the second and third stages. Also presents algorithm of recognition of types of road marking. At the end of the article, the results obtained are generalized, and further lines of research are given.
\end{abstract}

Keywords: autonomous car; computer vision; road detection; types of road marking detection; local extremes-points

\section{Introduction}

Currently, an increasing number of researchers agree that the use of autonomous cars will not only simplify and improve the safety of everyday life, but also fundamentally change the whole logic of urban infrastructure. [1] One of the most obvious consequence of creating an autonomous car is a dramatic increase in the level of road safety for both passengers and surrounding pedestrians. One of the probable consequence is the complete or partial disappearance of personal transport, the use of which will not be reasonable, since an autonomous car, when it is not used by the owner, can go into the taxi mode and bring its owner a passive income. Also, due to the fact the number of conventional cars are reduced to a minimum, the necessary parking space will be drastically reduced, which will partially save urban planners from building large parking facilities. [2] However, the technology that leads to such serious improvements of the human life quality, some of which was described above, is not completely solved, despite the huge number of specialists involved in its research. Also at the moment, there is no general approach to how this task should be solved, for example, despite the fact that building an autonomous car is a complex task, there is no unified understanding of the full set of subtasks to which it should be divided, but some basic subtasks can be identified. One of these basic subtasks is the recognition of the road marking, which naturally follows from the fact that the autonomous car is intended to move along city roads with the existing road marking, and in the process of movement it is required to ensure strictly following the traffic rules, in accordance with the existing marking lines. [3][4] This article is devoted to the solution of this problem. 


\section{Structure}

The main idea of this road marking recognition method is sequential filtering of image areas, which are fragments of the road marking with certain probability. The process of the markup recognition is divided into 4 consecutive stages: [5]

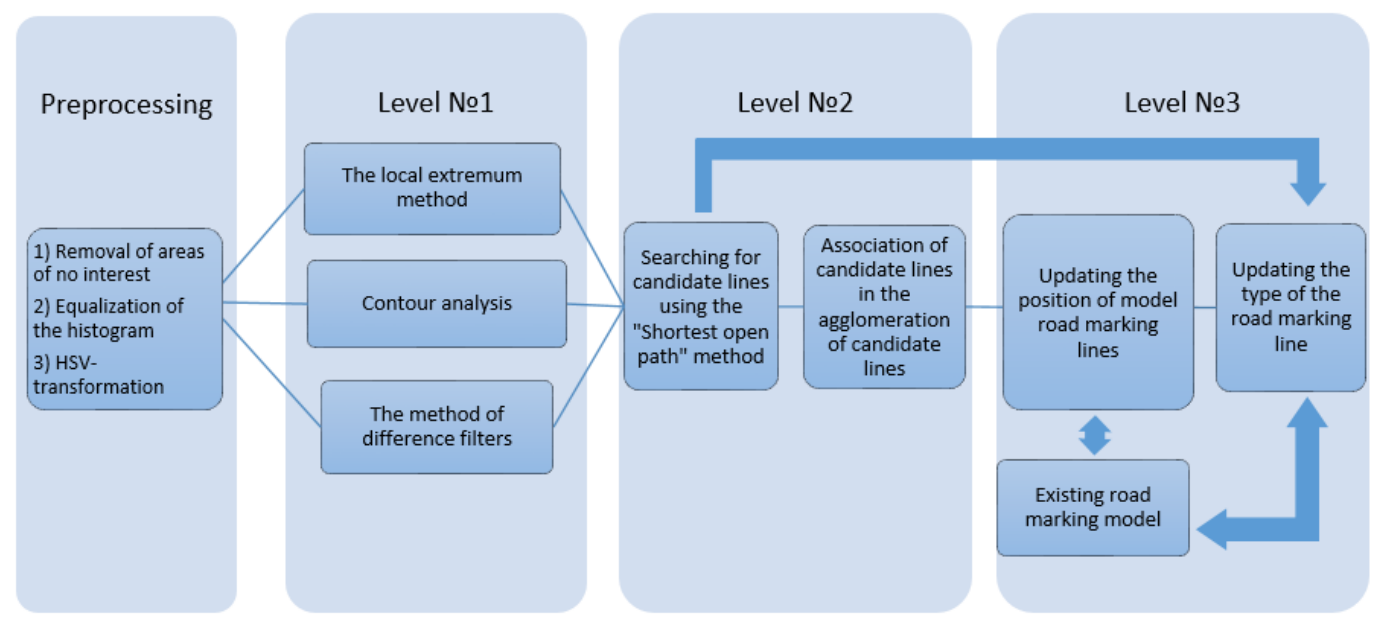

Fig. 1. Structure of the markup recognition process

\section{Pre-processing}

Immediately before recognition of the road marking, it is required to process the input image to reduce the amount of analysed information and bring the picture to a standardized view. Due to the fact that almost always the sky is in the field of view of the camera, it was decided to cut off the image area above the horizon line. To determine the level of the horizon line, the approximate location of the point is calculated, in which most of the straight lines or their extensions intersect due to the perspective effect.

\section{Search for the Level 1 primitives}

The first step in recognizing of the road marking is to search for image areas that are likely to be road marking elements with a certain probability. There are many methods to detect such sites, but after the analysis, authors conclude that it seems reasonable to use only three of them.

One such method is the local extremum method. Local extremes-points on the image have significant superiority of the brightness level over adjacent points, which can be considered as elements of the road marking. [3] The key idea of this method is the independent analysis of the small sections with a width of 1 pixel. Such areas are selected at a short distance from each other, evenly covering the entire image. For each site, a decision is made whether there is a road marking or not in the selected area. [6][7][8] An example of recognition of local extremes can be seen on Fig.2

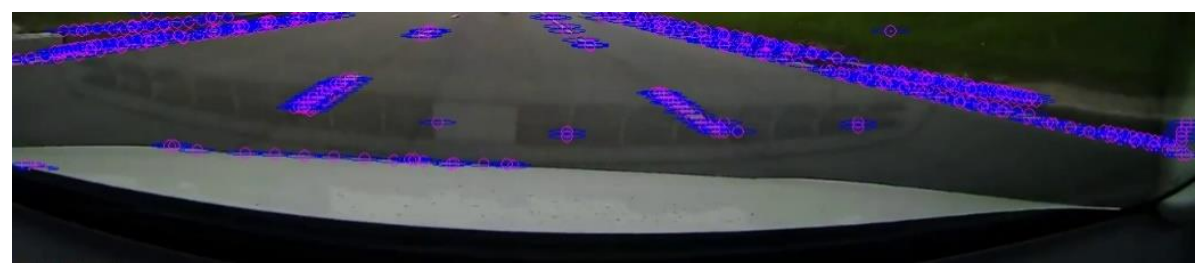

Fig. 2. Primitives of the first level - local extremes

The decision is made on the basis of the assumption that the brightness of the road marking significantly exceeds the brightness of the surrounding roadway. The scanning line is divided into 3 equal sections. The decision about the presence of the road marking in the area of the scanning line is made in case the sum of the brightness of the central section pixels is more than $\mathrm{K}$ times larger than the sum of the pixels of each side sections. Where $\mathrm{K}$ is the adjustable coefficient. Also, the analysis takes into account the absolute value of the colour H-component of the central region (HSV-colour space is used), which should lie in a certain range of values corresponding to the shades of white. This condition allows you to filter out sufficiently large percentage of the false positives of the first level detector, since the superiority of the brightness of the central section over the side ones can be observed at many objects not related to the road marking, such as leaves on a dark background, debris, etc. Another method of detecting primitives of the first level is the method of contour analysis. 
To find the outlines in the image, the Canny edge detector is applied, followed by the Hough transform to find the linear contours. This approach to the road marking detection is a very popular and stable method. However, the method allocates sections corresponding to the edges of the road marking line, and if the construction is performed based on the obtained sections, then each actual line will be recognized as two lines with different directions. Therefore, after finding the contours, the procedure of averaging the coordinates of all pairs of parallel lines lying at a distance of less than a certain predetermined threshold is required. An example of contour detection is shown on Fig.3.

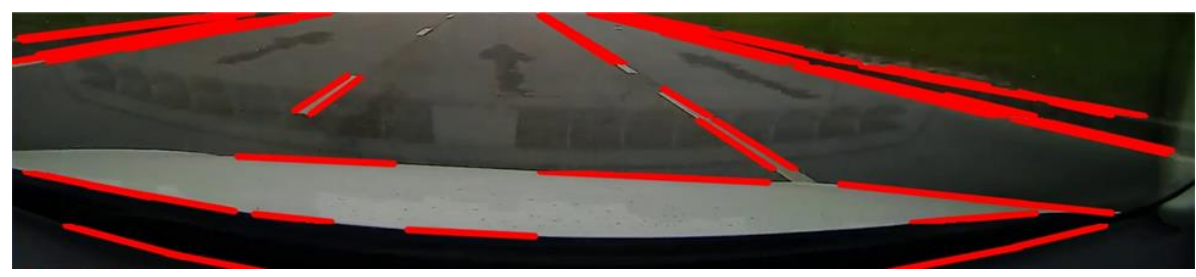

Fig. 3. Primitives of the first level, obtained by the method of contour analysis.

The last of the applied methods of detecting a primitive of the first level is a method based on the use of two line filters. The points in the image that have the largest difference between responses of these two line filters are considered road marking elements. The filters are given as follows:

$$
\begin{aligned}
& F_{1}=[-1,0,0 \ldots 0,2,0, \ldots,-1] \\
& F_{2}=[-1,0,0 \ldots 0,0,0, \ldots, 1]
\end{aligned}
$$

F1-filter determines the degree of burst intensity of the central pixel relative to the extreme pixels

F2-filter determines the degree of difference between the extreme pixels, i.e. The measure of the symmetry of the brightness distribution for the extreme zones of the investigated section.

The decision on whether or not the area to be surveyed belongs to the road marking is made by calculating the value:

$$
P=\left\{\begin{array}{l}
1, G_{1}-\left|G_{2}\right|>\text { Sensivity } \\
0, G_{1}-\left|G_{2}\right|<\text { Sensivity }
\end{array}\right.
$$

where G1 and G2 - Response of the first and second filters respectively

Sensivity - is a measure of the minimum required confidence in the results.

One of the advantages of these filters is their simple scalability, realized by changing the number of zeros in the matrix due to the symmetry with respect to the centre. An example of the image sections detection with the road marking by the difference filter method is shown on Fig.4.

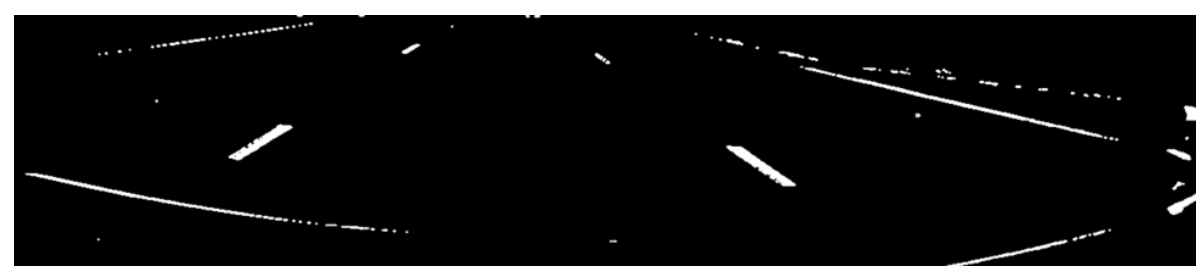

Fig. 4. Primitives of the first level, obtained by the method of difference filters.

After the detection of primitives of the 1-st level by all three methods, the results are merged into a single array.

\section{Search for the Level 2 primitives}

To ensure the model flexibility and at the same time maintain the simplicity of the mathematical description, the found array of primitives of the first level is divided into several horizontal sections. Each section is determined by the upper and lower pixel $Y$-coordinates-Ymin and $Y \max$ respectively. For each sector, all primitives of the first level with the $Y$ coordinate lying between -Ymin and Ymax are chosen. Moreover, the sections overlap each other in order to ensure more precise construction of the candidate line. It is assumed that the road marking line can be considered straight for each section. The construction of the candidate lines is performed for each site separately. Based on the first level primitives, the method of searching adjacent areas looks for clusters of the first level primitives with linear form. 
On the basis of each found cluster, an approximating candidate line is constructed, described by a linear equation. The lines found are taken for the so-called candidate lines. An example of constructing candidate lines is shown on Fig. 5.

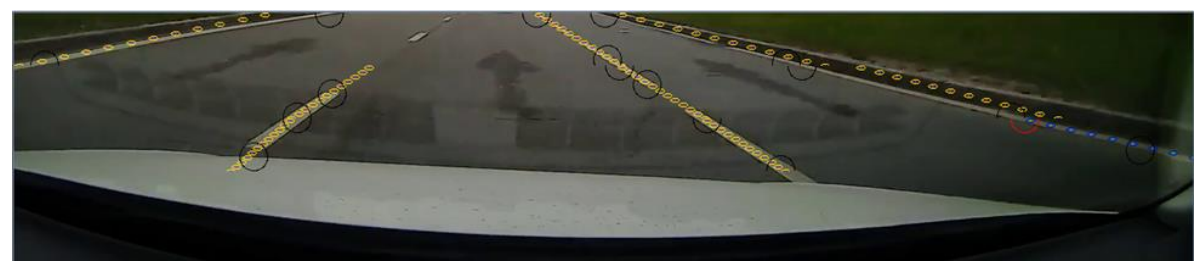

Fig. 5. Primitives of the second level-lines candidates.

Since the candidate lines consist of different parts of the image independently of each other, to obtain complete information about the road marking lines, candidates are merged forming agglomerations of the candidate lines. The junction points of the candidate lines on all frames have the same $\mathrm{Y}$-coordinates, since merging takes place on the lines which divided the image into sections. The conditions of the candidate lines merging are:

- The proximity of the lower and upper ends of the candidate lines of the upper and lower sections respectively

- The proximity of the slope coefficient of the candidates' joined lines.

These conditions can be defined as follows:

$$
\begin{aligned}
& \left|\mathrm{X}_{i}^{\text {down }}-X_{i+1}^{u p}\right|<P_{x} \\
& \left|K_{i}-K_{i+1}\right|<P_{k}
\end{aligned}
$$

Where $\mathrm{X}_{i}^{\text {down }}$ and $X_{i+1}^{u p}$ are the X-coordinate of the lower and upper ends of the $\mathrm{i}$-th candidate line $\mathrm{K}_{\mathrm{i}}$ - the slope coefficient of the $\mathrm{i}$-th candidate line

$\mathrm{P}_{\mathrm{x}}$ and $\mathrm{P}_{\mathrm{y}^{-}}$threshold values for the parameter $\mathrm{x}$ and $\mathrm{k}$ respectively. They determine the maximum possible difference of these parameters for the candidate lines being joined.

However, these conditions do not guarantee the uniqueness of the candidate line, because in some situations, candidates may be on the same level at a very short distance. In this case, it is required to decide which of the lines is more preferable for building a road marking model on its basis. Based on experimental results the most informative attribute is the length of the candidate line, i.e. the longer the candidate line, the more likely it can be said that this candidate line corresponds to the actual existing road marking line. In case of the candidate line absence, the agglomeration line is completed according to the assumption of linearity of the road marking. An example of constructing agglomerations of the candidate lines is shown on Figure 6.

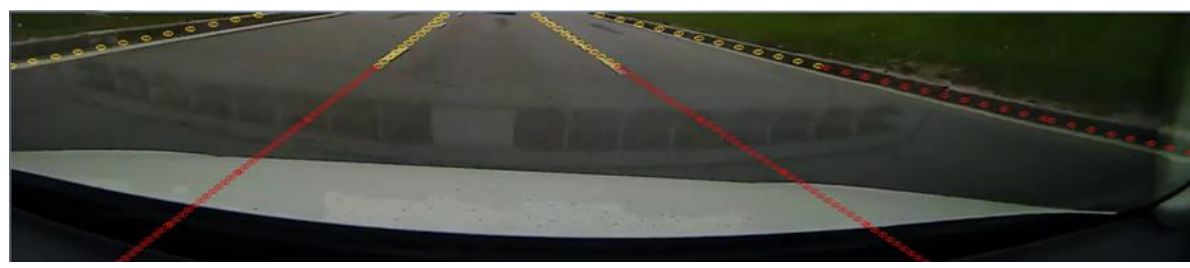

Fig. 6. Primitives of the second level-agglomeration of the candidate line.

\section{Search for Level 3 primitives - Model}

The final result of the work is the construction of a model describing the road marking located in front of the car view. The road marking model is a set of lines describing the real road marking lines reflecting their location and type. As well as agglomerations of the candidate lines, the model lines consist of straight segments rigidly tied to each other. During the movement, the existing model is adjusted due to new agglomerations of the candidate lines.

Algorithm for updating the position of the road marking model line

1. Obtain a new agglomerations of the candidate lines

2. Verify condition $\left|x_{i j}-x_{k j}\right|<p$

Where i-number of the existing model line

$\mathrm{j}$ - number of the joint between two segments

$\mathrm{k}$ - agglomeration number of the candidate line 
p-threshold value determining the maximum value of the road marking line offset between two frames based on the a priori knowledge of the car movement

3. If the condition is satisfied, the position of the model line is changed: $x_{i j}=x_{i j}+\left(x_{i j}-x_{k j}\right) * s p e e d \_L$ where speed_L is the coefficient indicating the level of confidence in the changes introduced by the agglomeration of the candidate lines.

After updating the position of the road marking lines, it is required to determine the type of the road marking line. The correct recognition of three types of road marking is implemented:

- Solid line

- Double solid line

- Intermittent line

Algorithm

1. Obtain an updated model road marking line

2. Capture the primitives of the first level on which basis this model line was formed

3. Determine the value of discontinuities between the captured primitives of the first level using skip $=\sum S$ where $S=\left\{\begin{array}{c}y_{i}-y_{i+1}, y_{i}-y_{i+1}>2 p \\ 0, y_{i}-y_{i+1}<2 p\end{array}\right.$

p- the search step for points of the first level $\mathrm{y}_{\mathrm{i}}$ - the $\mathrm{y}$ axis coordinates of the first level points

4. When the skip threshold is exceeded, the line is considered intermittent

5. Otherwise, the average density of points of the line is calculated as follows: density $=\frac{N}{L}$

Where N-quantity of primitives of the first level

L-line length

6. If the density threshold is exceeded, the line is considered to be double-solid

7. Otherwise, the line is considered single-solid

\section{Conclusion}

This article describes the basic steps and algorithms used to search for the road marking lines, which allow obtaining a stable recognition result in a wide range of external conditions, such as: illumination, weather, season. The main authors task today is to increase the number of the recognizable mark-up types, which is quite challenging problem, because the presence of any foreign objects on the road can form a non-existent gap in the road marking line, and errors in type recognition lines are not permissible, since the probability of violation of traffic rules significantly increases. Also, to automate the adjustment of the system to the changing conditions of the road environment, it is planned to use the methods of machine learning.

\section{Acknowledgements}

This research is supported by the Ministry of Education and Science of Russia. State assignment at Peter the Great St. Petersburg Polytechnic University (Department of «Mechatronics and Robotics»), project № 9.4040.2017/4.6.

\section{References}

[1] Cohen, A., Arce-Plevnik, L., \& Shor, T. (2016). IoT in automotive industry: Connecting cars. Unpublished paper.

[2] Dikmen, M., \& Burns, C. M. (2016). Autonomous driving in the real world: Experiences with tesla autopilot and summon. In Proceedings of the 8th International Conference on Automotive User Interfaces and Interactive Vehicular Applications, pp. 225-228. ACM.

[3] López, A., Serrat, J., Canero, C., Lumbreras, F., \& Graf, T. (2010). Robust lane markings detection and road geometry computation. International Journal of Automotive Technology, 11(3), pp. 395-407.

[4] Vacek, S., Schimmel, C., \& Dillmann, R. (2007). Road-marking Analysis for Autonomous Vehicle Guidance. EMCR.

[5] Weingerl, D., Kubinger, W., \& Engelhardt-Nowitzki, C. (2015). Preceding vehicle tracking in stereo images via 3D feature. Annals of DAAAM \& Proceedings, 26(1), pp. 997-1003.

[6] McCall, J. C., \& Trivedi, M. M. (2004, June). An integrated, robust approach to lane marking detection and lane tracking. In Intelligent Vehicles Symposium, 2004 IEEE, pp. 533-537, IEEE.

[7] Kammel, S., \& Pitzer, B. (2008, June). Lidar-based lane marker detection and mapping. In Intelligent Vehicles Symposium, 2008 IEEE, pp. 1137-1142, IEEE.

[8] Liu Y., Zuo L., Cheng T., Wang C. (2000). Development of an open parallel intelligent CNC milling system: Part 1, System structure, International Journal of Advanced Manufacturing Technology, Vol. 16, 537-541. 